\title{
Study on the Design of Avocado-fiber Skincare Knitted Underwear
}

\author{
Wang Shi*, Li Junjia and Zhao Fu \\ Dalian Polytechnic University
}

\begin{abstract}
With the continuous changes taken place in people's lifestyles, functional underwear has drawn attention from more consumer groups. Skincare, comfortable knitted underwear fabrics can be made by adding refined avocado oil into viscose to yield fibers and then blending it with other fibers such as acrylic and wormwood fiber. This article first introduces the moisture-retaining principles, raw material selection, and skincare property of avocado-fiber knitted fabric, and discusses the comfortability of the fabric, the requirements of underwear fitness, the style design optimization, and the functional area partitioning, aiming to provide design ideas for the development of the new skincare functional knitted underwear.
\end{abstract}

\section{Background}

With the rise of living standards, consumers are seeing a diversified demand for clothing and become increasingly demanding of functional fibers. Over the past few years, the beauty industry has developed rapidly, followed by the emergence of skincare fibers. By adding additives with cosmetic and skincare ingredients into fibers, researchers develop skincare fabrics and achieve the purpose of fulfilling both the wearability and beauty care results in fabrics. Due to its rich nutrients, avocado has been gradually gaining favor and popularity among health seekers. The extracted avocado oil boasts low acidity, non-irritation, and fine transdermal absorption. Therefore, apart from its edibility, avocado oil can also be used as a natural raw material for advanced skincare products and to make moisturizing skincare fibers that can adjust the skin conditions. Knitted fabric made of such fibers can meet the demand of female consumers for comfortability and skin beauty, and hence enjoys great market potential.

\section{The Development of Avocado-fiber Functional Knitted Fabric}

Traditional fall/winter underwear is often thermal solely, without taking into account the moisturizing need of skin. The dry and cold environment, especially in north China, can easily generate static electricity from frictions between the skin and clothes and between clothes and result in discomfort in wearing. On the other hand, wrapped in poorly ventilated, water-deficient underwear for a long while, human body will suffer burdens on surface skin and dry skin peeling, and harbor bacteria. So, the moisturizing effect of fall/winter underwear fabric turns out to be a key factor to improve the wearing experience.

\subsection{The Moisture-retaining Principles of Avocado Fiber}

The main constituent elements of human sebum are fatty acids, one of which is oleic acid. A major cause for dry skin is the poorly-moisturizing stratum corneum and the moisture imbalances. Oleic acid is a great moisturizer that can soften keratin and maintain skin sebum resilience. Avocado oil is rich in oleic acid and palmitoleic acid that can condition the skin environment. It also contains rich anti-aging vitamin E, which can gently protect the skin, make it smoother, and effectively relieve skin dryness in winter.

\subsection{Selection of Raw Materials and Blending Composition}

The development of fall/winter knitted underwear fabrics should be based on the consideration of good thermal protection, so acrylic fiber is chosen as the main blending material. Acrylic fiber, also known as "artificial wool", shares similar properties with wool, but its warmth retention, elasticity and strength are all better than wool, and it is also quite soft. Second, the fabric should be somewhat antibacterial, so wormwood fiber is added. Wormwood fiber, made by adding nano-sized wormwood leaf powder into viscose fiber, is antibacterial and deodorant, and can improve the skin environment and promote the skin surface health. The third fiber is avocado fiber, which can provide important moisturizing and skincare effects. Finally, a small amount of modal and spandex blends is added to increase moisture absorption and elasticity of the fabric and improve its comfortability and durability. The specific composition

\footnotetext{
*wangshi@dlpu.edu.cn
} 
is: acrylic fiber $50.6 \%$, wormwood fiber $18.4 \%$, avocado fiber $13.8 \%$, modal $9.2 \%$, and spandex $8 \%$; and the fabric weight is $220 \mathrm{~g} / \mathrm{m} 2$.

\subsection{Fabric Performance}

The fabric is basically weaved with flat weft-knitted, single-sided stitches by circular knitting machines.
Besides its long-lasting moisture retention and smooth skin-friendly feel, it also boasts great moisture absorption and breathability, with a moisture absorption rate that is 1.7 times that of ordinary like products. It is also thermal and antimicrobial (see Table 1). With such an excellent comprehensive performance, this fabric is ideally suited for the development of fall/winter underwear.

Table 1. Test Results of The Fabric's Moisture Absorption Rate \& Comfortability

\begin{tabular}{|c|c|c|c|}
\hline \multirow[t]{2}{*}{ Condition } & \multirow[t]{2}{*}{ Time/h } & \multicolumn{2}{|c|}{$\begin{array}{c}\text { Moisture Absorption } \\
\text { Rate }(\%)\end{array}$} \\
\hline & & $\begin{array}{l}\text { Original } \\
\text { Sample }\end{array}$ & $\begin{array}{l}\text { Contrast } \\
\text { Sample }\end{array}$ \\
\hline \multirow{6}{*}{$\begin{array}{c}40^{\circ} \mathrm{C} \\
\times 90 \% \mathrm{RH}\end{array}$} & 0 & 0 & 0 \\
\hline & 1 & 7.4 & 4.4 \\
\hline & 3 & 9.9 & 5.7 \\
\hline & 5 & 10.6 & 6.1 \\
\hline & 7 & 10.9 & 6.4 \\
\hline & 24 & 11.4 & 6.6 \\
\hline \multirow{5}{*}{$\begin{array}{c}20^{\circ} \mathrm{C} \\
\times 65 \% \mathrm{RH}\end{array}$} & 25 & 10.2 & 5.7 \\
\hline & 27 & 8.8 & 5.4 \\
\hline & 29 & 8.5 & 5.1 \\
\hline & 31 & 8.4 & 4.9 \\
\hline & 48 & 8 & 4.5 \\
\hline
\end{tabular}



\section{Analysis of Design Factors of Skincare Functional Underwear}

In developing functional clothing, it is necessary to position the product accurately, that is, which consumer group the product is targeted at and what functional and performance requirements it should meet. The development of functional clothing should adapt to the current economy and people's demand for material culture. Different consumer groups have diverse ideas on consumption and functionality.

\subsection{Target Customer}

Avocado-fiber functional knitted underwear features great care for the skin, which coincides naturally with females' consumption preferences. With the improvement of living standards, women attach importance to not only facial care but also body care. Body lotions containing whitening and scrubbing agents

have become a must-have for most women, revealing females' enthusiasm for body beauty. Therefore, it's very important to fully display the product's functionality. On the other hand, female consumers who prefer skincare functional knitted underwear are not only concerned about the product quality, but also tend to set higher expectations for beauty, which not only entails the exterior design of product but also its skincare effect.

\subsection{Fabric Comfortability}

Underwear is the clothing that directly touches human skin, so its comfortability turns out to be fairly important. The knitted fabric is a suitably resilient material that can adapt to the flexing, bending and twisting of human body. Its fine breathability can also keep fluffy and soft even when the skin is well moisturized.

\subsection{Fitness}


The knitted underwear fabric developed based on avocado fiber features skin moisturizing, so the product should fit the skin as much as possible to give full play to the fabric's moisturizing effect. Female body curves are mainly shown in their breast, waist and hips and the undulation of muscles. Traditional knitted underwear rarely has split lines, so it is quite loose and fails to fit the

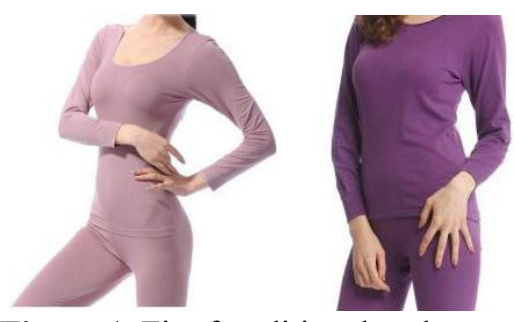

Figure 1 Fit of traditional underwear

\subsection{Factors of Style Design}

Ordinary knitted underwear on the market is often linear and lacks a targeted body-fitting structure. It is plane while draped for hanging, so both its wearing effect and fitness are far from satisfactory. To improve moisture retention, the product should comprise targeted splits and splices at the breast, waist, hips and the undulating parts of muscles to showcase the overall curve shape and maintain its modelling when it is not worn, so that it can fit the female body better during wear. body well, especially under the breast and arms, and at wrists and legs (see Figure 1). Skintight sportswear usually fits the body ideally (see Figure 2). The fitness of knitted underwear developed based on the moisture retention of avocado fiber should lie somewhere between traditional knitted underwear and skintight sportswear, so that it can serve better moisture retention and skincare.



Figure 2 Ideal fit of functional underwear

For example, as shown in Figure 3, Style A contains an arc-shaped plane splits at breast and hips to highlight its solid structure. Its V-shaped split line on the abdomen not only exhibits a balance of the body volume, but also visually makes the waist look slenderer. Furthermore, the split lines on the back and limbs also fit well with the muscle undulations to enhance the wearing comfortability and visually lift the body up. In Style B, proper pleats are added on the breast to highlight its modeling and well fit the body at the lower part of breast; the split lines at waist and back are also functional and nice-looking; and for the underpants, the small pieces of splices at the raised parts on the legs have further enhanced the fitness.



Figure 3 Underwear design reference

\subsection{Functional Area Partitioning}

The development of functional underwear should be based on reasonable structural division and design targeted at different parts' functional requirements. For example, for underarm areas, moisture retention is not needed, but smell-removing and germicidal functional fabrics are much preferred. Second, the warm-keeping demand of the waist and abdomen. Both men and woman are likely to get a chill on these areas, so the thermal effect should be accordingly strengthened, and pile stitches with better heat preservation should be selected. In addition, considering the functionality of body beauty, fabrics on the inner thigh and the outer calf should be thicker to put a certain pressure on the skin, tighten the figure, and thus achieve body-shaping effect.

\section{Conclusion}

What counts most in the design of avocado-fiber knitted underwear is to realize its moisturizing function, wearing comfortability and overall aesthetics. The factor that influences the moisturizing effect is the underwear's fitness with the skin. To improve such fitness, it's necessary to analyze thoroughly the relationship between clothing and human body and design "people-oriented" products. Innovative design of style and pattern is an important solution. On the other hand, comfortability is an essential requirement of underwear. The selection of fabrics and the partitioning of human body's functional areas are major factors to improve comfortability. Ultimately, with functionality and comfortability fulfilled, 
aesthetics should also be integrated into product design to meet female consumers' propensity to beautify themselves and cater to the market demand.

\section{References}

1. Lv Ning, Gao Wenjuan. Research Progress of New Skin Care Fiber[J]. Melliand China, 2016, 44(05): 16-18 \& 20.

2. Matcha, Mustard. A Popular Food-grade Cosmetic Raw Material: The Application of Shea Butter in Cosmetics[J]. China Cosmetics Review, 2018 (12): 56-59.

3. Wang Yuhong, Wang Sujuan, Zhao Lina, \& Liu Xingkun. Research and Development of Functional Clothing[J]. Shandong Textile Economy, 2020 (02): 10-12.

4. Zhang Yingli. The Clothing for Cosmetic Mask A New Experience of Skin Care[J]. Progress in Textile Science \& Technology, 2010 (04): 4-5 \& 9.

5. Zhou Yi, Chen Fanliang. The Design Ethics and Development Trends of Female Underwear[J]. West Leather, 2020, 42 (01): 82.

6. Li Yuanyuan. Aesthetic Culture Research on the Current Female Body Consumption in China[D]. Northwest University for Nationalities, 2019.

7. Hu Jun. Children's Smart Watch is not Omnipotent for Anti-Abduction [N]. China Consumer Journal, 2015-07-30 (001).

8. Bai Rui, Chen Linyun, \& Wu Yufeng, \& Huang Xiaoyun. Development of Camellia Oil Fiber Knitted Functional Underwear Fabric[J]. Knitting Industries, 2020 (04): 5-8. 